\title{
Swansong Biospheres: The biosignatures of inhabited earth-like planets nearing the end of their habitable lifetimes
}

\author{
Jack T. O'Malley-James ${ }^{1}$, Jane S. Greaves ${ }^{1}$, John A. Raven ${ }^{2}$ \\ and Charles S. Cockell ${ }^{3}$ \\ ${ }^{1}$ School of Physics \& Astronomy, University of St Andrews, St Andrews, UK \\ email: jto5@st-andrews.ac.uk \\ ${ }^{2}$ Division of Plant Sciences, University of Dundee at TJHI, The James Hutton Institute, \\ Invergowrie, Dundee, UK \\ email: j.a.raven@dundee.ac.uk \\ ${ }^{3}$ UK Centre for Astrobiology, School of Physics \& Astronomy, University of Edinburgh, \\ Edinburgh, UK \\ email: c.s.cockell@ed.ac.uk
}

\begin{abstract}
The biosignatures of life on Earth are not fixed, but change with time as environmental conditions change and life living within those environments adapts to the new conditions. A latitude-based climate model, incorporating orbital parameter variations, was used to simulate conditions on the far-future Earth as the Sun enters the late main sequence. Over time, conditions increasingly favour a unicellular microbial biosphere, which can persist for a maximum of 2.8 Gyr from present. The biosignature changes associated with the likely biosphere changes are evaluated using a biosphere-atmosphere gas exchange model and their detectability is discussed. As future Earth-like exoplanet discoveries could be habitable planets nearing the end of their habitable lifetimes, this helps inform the search for the signatures of life beyond Earth
\end{abstract}

Keywords. Astrobiology, Sun: evolution, Earth.

\section{Introduction}

The increasing luminosity of the Sun as it ages will cause surface temperatures on Earth to increase, eventually leading to runaway ocean evaporation, making conditions more favourable for a unicellular microbial biosphere, similar to (but smaller than) that present for the first 2.5 Gyr of Earth's habitable lifetime (O'Malley-James et al. 2013a).

\section{Temperature Model}

The primary driver for habitability change is the solar luminosity increase over time, which can be modelled using the methods of Gough (1981), which, when combined with future orbital parameter changes, then determines surface insolation. This, coupled with greenhouse gas changes is used to calculate the flux of energy in and out $\left(F_{\text {in } / \text { out }}\right)$ of the Earth climate system,

$$
\frac{d T}{d t}=\frac{F_{\text {in }}-F_{\text {out }}}{C_{p}}
$$

at different latitudes (where $C_{p}$ is the heat capacity at constant pressure), giving a mean surface temperature profile for the planet. The temperature change with altitude can also be derived from this by calculating the adiabatic lapse rate. 


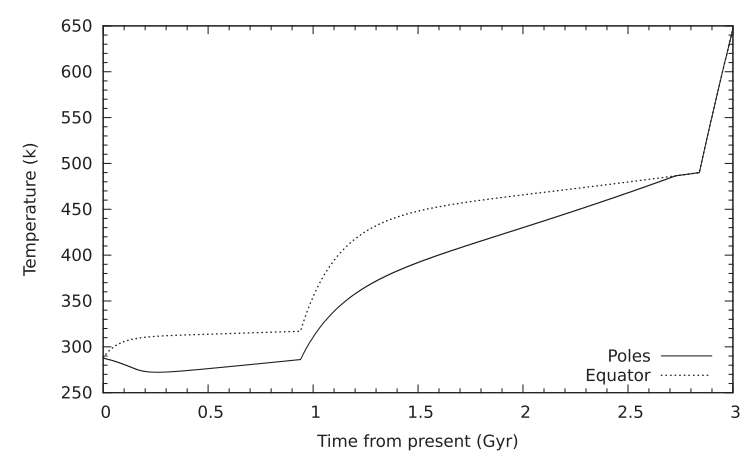

Figure 1. Change in global mean temperature over time with increasing solar luminosity. (O'Malley-James et al. 2013a).

\section{Stages of biosphere death}

Increased temperatures cause an increase in atmospheric water vapour, raising silicate weathering rates, which draws down more atmospheric $\mathrm{CO}_{2}$. Decreasing $\mathrm{CO}_{2}$ levels result in the gradual decline of plant species and the animal species they depend on (Calderia \& Kasting, 1992; O'Malley-James et al. 2013a;b). This leaves behind a microbial biosphere.

Rapid ocean evaporation begins 1 Gyr from present. As water vapour is a greenhouse gas, there is a rapid increase in surface temperatures, reducing the extent of the biosphere by restricting life to remaining areas of liquid water. Such refuges include high altitude pools (for 2.2 Gyr from present), cold trap caves (for 2.8 Gyr from present) and the subsurface (O'Malley-James et al. 2013a).

The atmosphere-biosphere model is driven by the surface temperature model. For rapid extinction rates, the die-off of plants and animals may be indicated by elevated atmospheric $\mathrm{CH}_{4}$ levels, isoprene and ethane (O'Malley-James et al. 2013b). Unlike the more productive microbial biospheres of the early Earth, the only potentially detectable biosignature of a far-future microbial biosphere is $\mathrm{CH}_{4}$, although this may not reach remotely detectable levels in the atmosphere, essentially making a dying biosphere undetectable.

\section{Summary and Conclusions}

As Earth nears the end of its habitable lifetime it will only be capable of supporting microbial life adapted to multiple extreme conditions. This makes Earth a world predominantly inhabited by microorganisms for approximately two-thirds of its habitable lifetime, which, assuming a similar evolutionary history on Earth-like exoplanets, makes microbial biosignatures important in the search for life beyond the solar system.

\section{References}

Caldeira, C. \& Kasting, J. F.: The lifespan of the biosphere revisited, Nature, Vol. 360, pp. 721-723, 1992.

Gough, D. O.: Solar interior structure and luminosity variations, Solar Phys., Vol. 74, pp. 21-34, 1981.

O’Malley-James, J. T., Cockell C. S., Greaves, J. S., \& Raven, J. A.: Swansong Biospheres II: The final signs of life on terrestrial planets near the end of their habitable lifetimes, 2013b, arXiv: 1310.4841

O’Malley-James, J. T., Greaves, J. A., Raven, J. A., \& Cockell, C. S.: Swansong Biospheres: refuges for life and novel microbial biospheres on terrestrial planets near the end of their habitable lifetimes, Int. J. Astrobiology, Vol. 12, pp. 99-112, 2013a. 